\title{
Prototype of a Zero-Field-Cooled YBCO Bearing With Continuous Ring Permanent Magnets
}

\author{
António J. Arsénio (1), Miguel Roque (1), Carlos Cardeira (), Member, IEEE, \\ Paulo J. Costa Branco (), Senior Member, IEEE, and Rui Melicio ${ }^{(0)}$, Senior Member, IEEE
}

\begin{abstract}
Based on the levitating design of the Zero-FieldCooled (ZFC) Maglev previously developed, a novel horizontal ZFC superconductor magnetic bearing (SMB) is currently being developed. However, simulations have shown that its stator and rotor made of YBCO bulks and $\mathrm{NdFeB}$ permanent magnets (PM), respectively, present significant magnetic discontinuities. These produce asymmetries on the bearing rotation and several stable and unstable position angles that prevent the SMB from rotating smoothly, leading to a step motor like sort of rotation. Those drawbacks are now avoided by using ring shape PMs, leading not only to a smooth rotation of the SMB but also increasing its stability. New three-dimensional finite element method simulations and a set of experimental tests with the new SMB show in this paper the viability and increased performance when ring shape PMs are used. Finally, experimental results using the prototype also show that the model fits very well with the laboratory test results.
\end{abstract}

Index Terms-High-temperature superconductors, bearings, levitation, YBCO, rotating machines.

\section{INTRODUCTION}

I $\mathrm{N}$ PREVIOUS work, our research team achieved reliable results using superconductor technology to create and study levitation systems, namely a new Maglev design using ZFC YBCO superconductors [1], [2], among others. Following similar concepts and strategies, a ZFC YBCO magnetic bearing has been developed [3]-[5], using PMs and type II superconductor bulks. Lately, most of the existing levitation systems use these two components; and regarding levitating bearings and flywheels, their application is expected to increase. In [6] a passive SMB was investigated to create a more effective twist element in the textile technological process of ring spinning since frictional wear and heat are the limiting factors for the productivity of such devices. A multi-surface levitation concept using three PMs and one HTS is studied for different configurations in [7], to reach the highest possible levitation forces in order to maximize the stiffness of a mechanical flywheel system. In [8] an HTS bearing was tested using ZFC and Field Cooling

Manuscript received September 19, 2017; accepted March 6, 2018. Date of publication March 22, 2018; date of current version April 10, 2018. (Corresponding author: António J. Arsénio.)

A. J. Arsénio, M. Roque, C. Cardeira, and P. J. Costa Branco are with the IDMEC, Instituto Superior Técnico, Universidade de Lisboa, Lisbon 1649-004, Portugal (e-mail: antoniojcosta@ ecnico.ulisboa.pt).

R. Melicio is with the IDMEC, Instituto Superior Técnico, Universidade de Lisboa, Departamento de Física, Escola de Ciências e Tecnologia, ICT, Universidade de Évora, Évora 7000, Portugal.

Color versions of one or more of the figures in this paper are available online at http://ieeexplore.ieee.org.

Digital Object Identifier 10.1109/TASC.2018.2817279

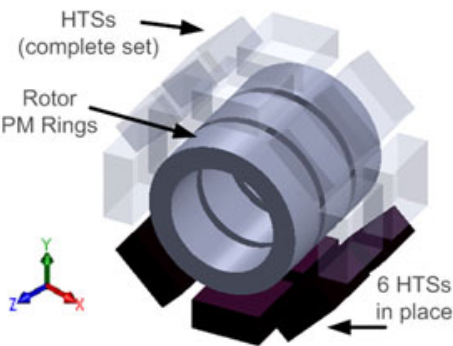

(a)

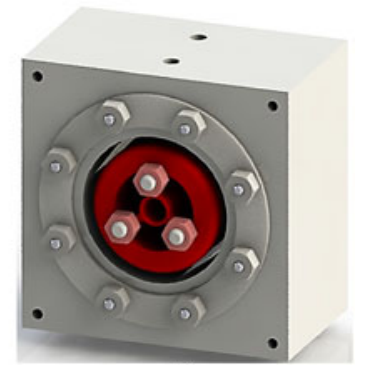

(b)
Fig. 1. Schematic of ZFC-SMB: (a) spatial distribution of PMs and HTS bulks, (b) final assembly.

(FC). The results of both techniques were compared, concerning axial forces, axial stiffness, and their characteristics. Other authors have deeply studied important features of SMB, namely dynamical stiffness models [9] and effect of the magnetic configuration on the rotational motion [10]. However, most of the studied levitation systems use FC to cool the HTSs. ZFC reveals some advantages when compared with the field cooling technique because FC implies significant hysteresis losses due to magnetic flux trapping [1], [2], [11]. Furthermore, for horizontal SMB, previous studies showed that Zero Field Cooling provides more effective lateral stability and higher levitation forces than the commonly used FC [5]. Based on such principles, an original ZFC-SMB was then designed and produced. The previously reported ZFC-SMB [3]-[5] was composed of a fixed part and a rotating but levitating part, respectively stator and rotor, as shown in Fig. 1(a) and (b). The stator contains two discontinuous rings. Each discontinuous ring is composed of eight equally spaced bulk YBCO superconductors as presented in Fig. 1(b). Each bulk has a rectangular shape with dimensions of $32 \times 32 \times 14 \mathrm{~mm}$. The YBCO bulks are produced with melttextured $\mathrm{YBa}_{2} \mathrm{Cu}_{3} \mathrm{O}_{7-\mathrm{x}}$ with $\mathrm{Y}_{2} \mathrm{BaCuO}_{5}$ excess, prepared by seeded melt growth method, with a critical temperature around $90 \mathrm{~K}$ [12].

The rotor part contains three discontinuous rings each of five equally spaced NdFeB PMs. Each PM $\left(B_{r}=1.25 \mathrm{~T}\right)$ has a rectangular form with dimensions of $25 \times 25 \times 12 \mathrm{~mm}$. The three inner discontinuous rings of PMs are magnetized in an alternating North-South-North configuration, such that the two outer rings of magnets have concordant polarizations and the middle ring of magnets opposite polarization [3]-[5].

Due to budget limitations, only six superconductors were used on the bottom of the stator in the prototype version. The six 

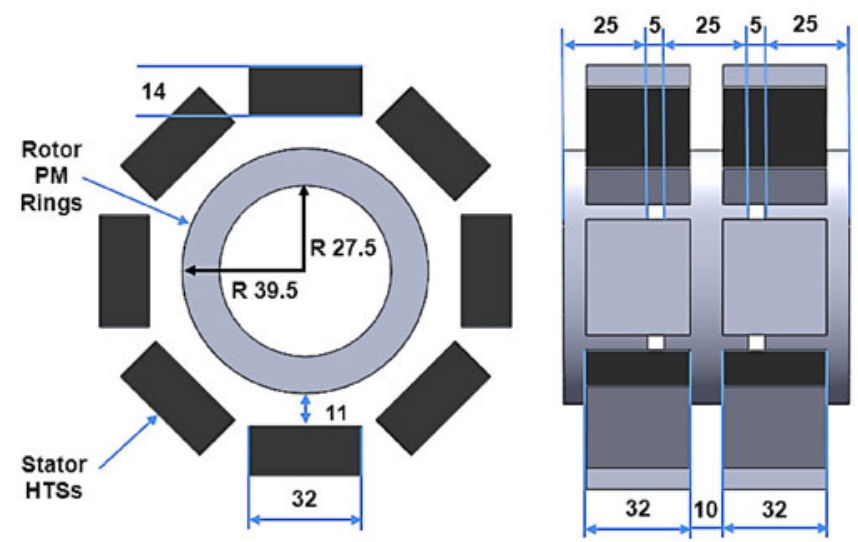

Fig. 2. Rotor D5 geometry and dimensions of the new SMB design.

$(2 \times 3)$ superconductor bulks position is shown in Fig. 1(a). Simulations and experimental tests show that the rotor and stator with such a design present discontinuities that cause asymmetries in the rotation and several stable and unstable angles that prevent the SMB to rotate smoothly.

In this paper, the discontinuous PMs used previously are replaced by three $\mathrm{NdFeB}$ radially magnetized ring $\mathrm{PMs}$ with grade $\mathrm{N} 40\left(B_{r}=1.25 \mathrm{~T}\right)$. These magnets have similar features, when compared with the previously layout of the PMs. However, due to manufacturing limitations, there are some differences. The outer radius is $39.5 \mathrm{~mm}$ instead of $41.5 \mathrm{~mm}$, whilst the inner radius is $27.5 \mathrm{~mm}$ instead of $29.5 \mathrm{~mm}$. The main concern was to keep the thickness of $12 \mathrm{~mm}$. The width remains $25 \mathrm{~mm}$ as well. The weight of the three rings together is $1.5 \mathrm{~kg}$. The whole structure is kept symmetrical for stability reasons. The conception and modelling of the new rotor is presented and the improvements and developments of the ZFC-SMB are analyzed.

In this paper the following terminology will be used:

- Lifting forces: sustaining forces that keep the rotor levitating;

- Guidance forces: horizontal forces that push the rotor to the center;

- Rotor vertical offset: rotor vertical displacement from the center;

- Rotor horizontal offset: rotor horizontal displacement from the center.

\section{MODELLING}

The detailed geometry and dimensions of the new SMB design are shown in Fig. 2. The three-ring PMs are magnetized in an alternating North-South-North configuration. The distance between each ring defines the type of rotor and it is important since lifting and guidance forces vary with it. Three different rotors are studied: D5, D10, and D20, which means $5 \mathrm{~mm}, 10 \mathrm{~mm}$ or $20 \mathrm{~mm}$ of distance between each PM. Fig. 2 represents rotor D5.

\section{A. Simulation of the Magnetic Flux Densities}

Figs. 3 and 4 represent simulated magnetic flux density in the SMB with six yttrium barium copper oxide (YBCO) bulks in

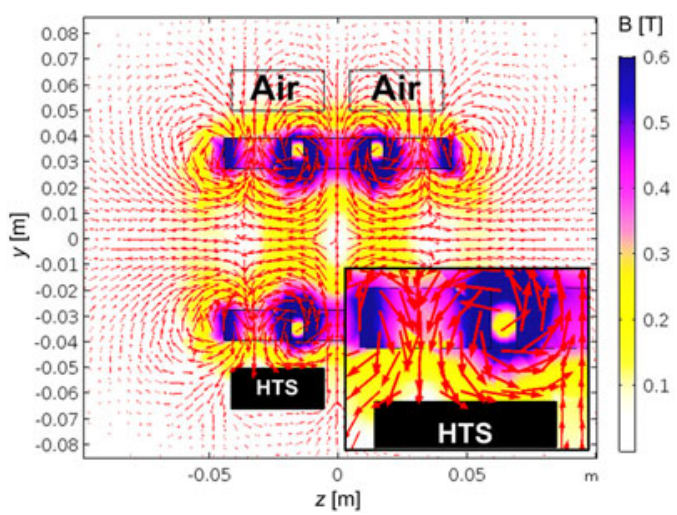

Fig. 3. Simulation of magnetic flux densities with rotor D5 along the YZ plane.

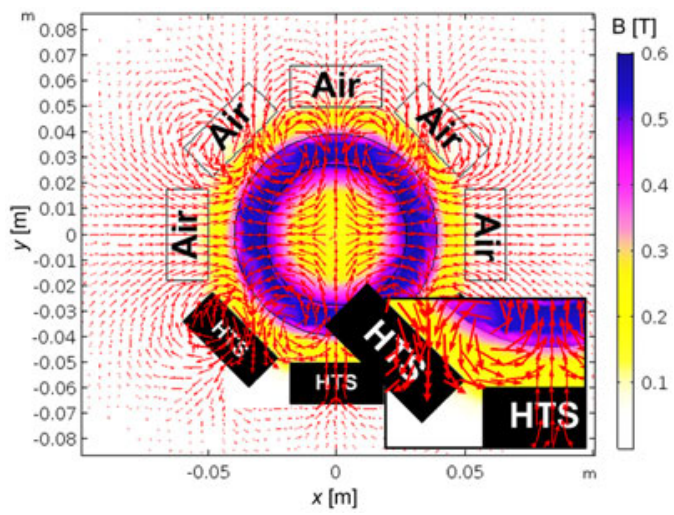

Fig. 4. Simulation of magnetic flux densities with rotor D5 along the XY plane.

stator and ring PMs in the rotor. A 3D Finite Element Method (FEM) model was obtained assuming a relative magnetic permeability of $\mu_{r}=0.2$, as explained in [3]. This approach cannot be used for computation of Joule losses and the associated temperature increase in the superconductors. However, this simple model allows the proposed SMB design to be tested without excessive computational effort due to its $3 \mathrm{D}$ representation and is sufficient to produce reliable quantitative results [3]. In both figures, a zoom of the region close to PMs and HTS Bulks is presented to show the magnetic flux in this region.

The radial component of magnetic induction reduces in the direction of the six YBCO bulks under the rotor because they present a relative permeability of 0.2 .

\section{B. Prediction of Lifting and Guidance Forces}

Fig. 5 shows lifting forces vs. rotor vertical offset obtained from the 3D FEM model [3], for the three different rotors. Results were obtained for decentering the rotors over a range of $\pm 7 \mathrm{~mm}$, which represents the possible axial displacement of the rotor into the stator. On the other hand, guidance forces are shown in Fig. 6. Displacement zero represents the stable position, in which the rotor is centered and has no external forces applied. 


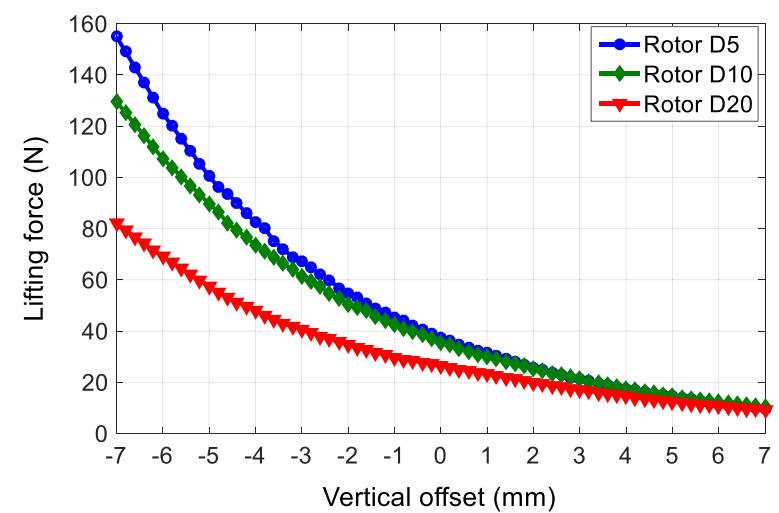

Fig. 5. Lifting rotor forces for different rotor vertical offset values. Results for the three different rotors are shown.

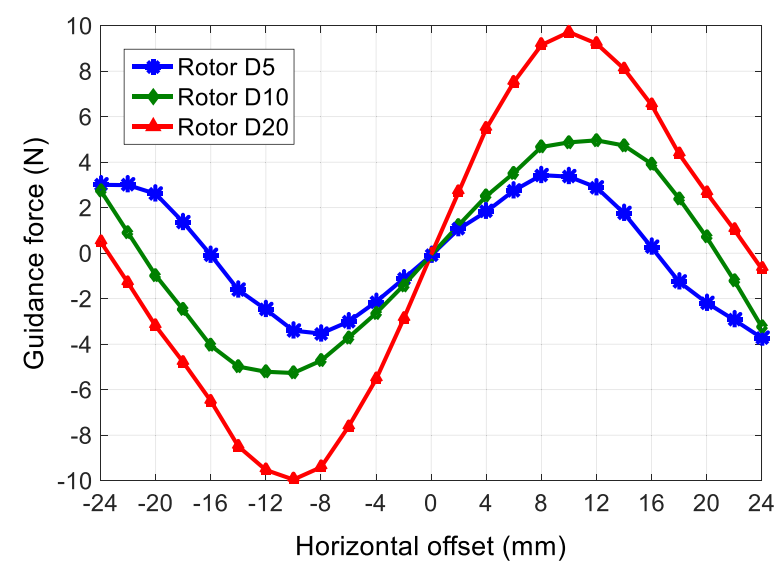

Fig. 6. Guidance rotor forces for different horizontal offsets. Results for the three different rotors are shown.

TABLE I

LIFTING AND GUIDANCE FORCES FOR DIFFERENT ROTOR DIMENSIONS

\begin{tabular}{lcc}
\hline \hline Rotor type & Lifting force centered rotor & Maximum guidance forces \\
\hline D5 & $36.9 \mathrm{~N}$ & $3.53 \mathrm{~N}$ \\
D10 & $34.8 \mathrm{~N}$ & $5.27 \mathrm{~N}$ \\
D20 & $26.5 \mathrm{~N}$ & $9.72 \mathrm{~N}$ \\
\hline \hline
\end{tabular}

Table I shows lifting and maximum guidance forces with the rotor centered, for different rotor dimensions.

As one can see, guidance forces increase as the distance between the PMs increases, whereas the lifting forces decrease.

\section{PRototype DeSIGN}

The SMB stator was not modified. All the changes in the prototype are present in the SMB rotor. A 3D CAD design was again used for its conception. The stator keeps the same configuration as in [3]-[5] with YBCO superconductor bulks. See [3]-[5] for more detailed information about the SMB stator.

The rotor must reveal circular geometry and it has to support the PMs as presented in Fig. 2. Further requirements are: i) keep the distances between each ring PM and between HTSs

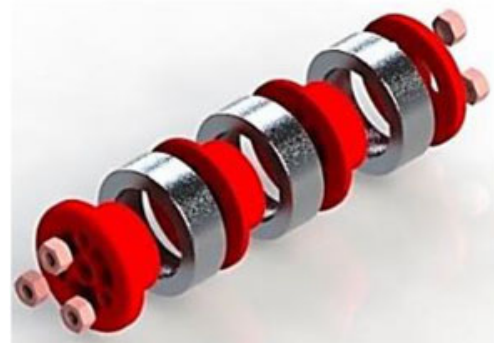

(a)

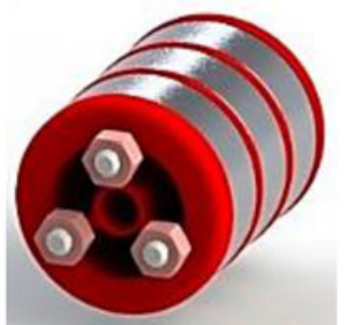

(b)
Fig. 7. Rotor: a) Exploded view. b) Assembled view.

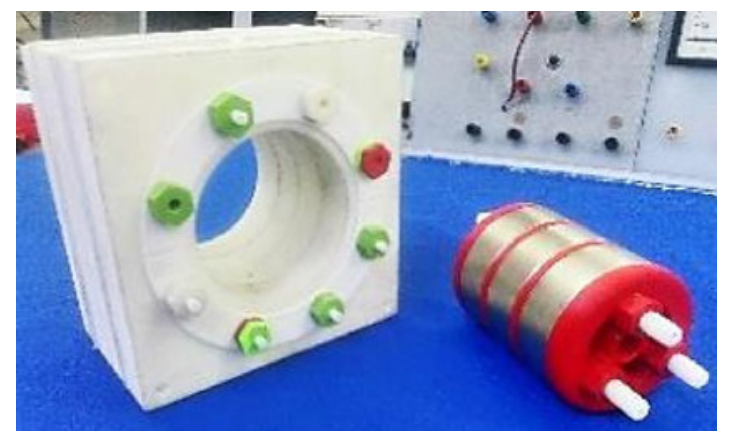

Fig. 8. Real prototype with rotor D5.

and PMs (air-gap); ii) allow an easy and practical assembly and disassembly, in such a way that the PMs damage risks are as minimal as possible; iii) be as light as possible, ensuring the stiffness of the structure; iv) develop a modular prototype.

Four main parts were designed to fulfill the requirements of the structure: two internal spacers to keep the intended distances between each PM and two external caps. Three threaded rods are used to maintain the structure compact and six nuts are used to assemble both stator and rotor together.

Fig. 7(a) shows the rotor exploded view with two internal slices, two external slices (base and cap) and six nuts, besides the three-ring PMs. Fig. 7(b) shows the rotor assembly.

The real prototype was built using a 3D printer. The chosen materials have a relative magnetic permeability of about 1 . The rotor slices and the nuts are made of Polylactic Acid (PLA) in order to have high resistance and good finishing, whilst Teflon (polytetrafluoroethylene) was used for the rods, to ensure better finishing after machining the threads. For electrical and mechanical properties of these materials see [13], [14]. The real prototype of the rotor D5 and the stator is presented in Fig. 8. There is no special casing for the SMB besides the stator slices.

The measured mass of rotors D5 and D20 are $1.57 \mathrm{~kg}$ and $1.62 \mathrm{~kg}$, respectively.

Several tests were made to prove the stiffness of the structure and the ease and security with which the assembly could be mounted and dismounted.

\section{MEASUREMENT OF LifTing AND GuidANCE ForCES}

All the tests were made after cooling the YBCO bulks in the absence of magnetic field, i.e., following the $\mathrm{ZFC}$ technique. 


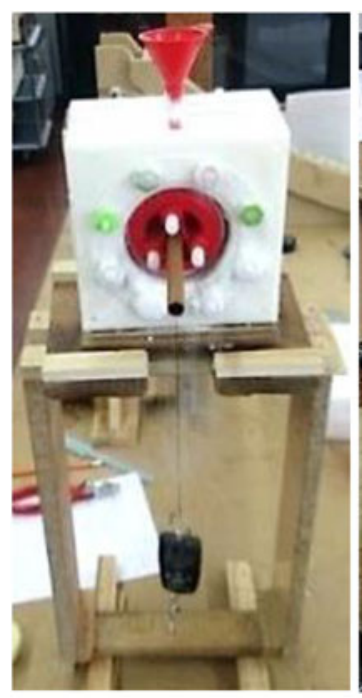

(a)

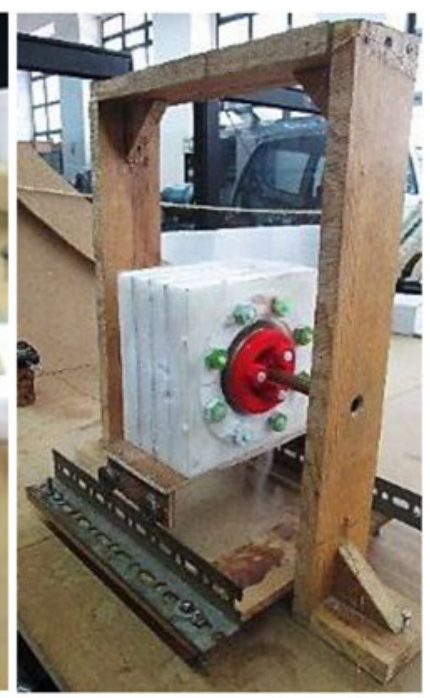

(b)
Fig. 9. Experimental setups to measure: a) Lifting forces, b) Guidance forces.

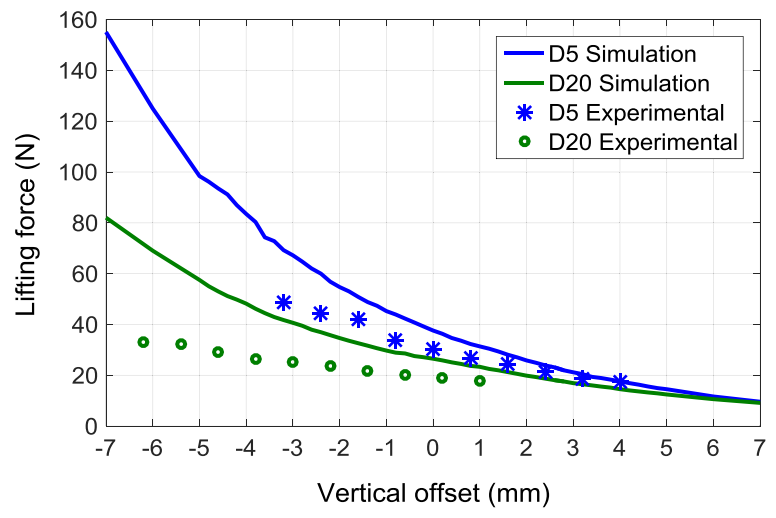

Fig. 10. Rotor lifting forces versus vertical offset.

The SMB stator was very slowly submerged in a liquid $\mathrm{N}_{2}$ bath. After around $30 \mathrm{~min}$, when the stator was all submerged and when all the $\mathrm{N}_{2}$ bubbles had disappeared, the stator was removed and placed with the rotor on the motor shaft. The cooling was maintained by pouring liquid $\mathrm{N}_{2}$ into the stator as shown in Fig. 9(a).

Fig. 9(a) shows the experimental setup used to measure levitating forces. The rotor stays lifting, whilst the lifting force is measured by the force sensor. The rotor and the sensor are linked by a copper wire. This material was chosen, due to its high modulus of elasticity.

Fig. 9(b) shows the experimental setup used to measure guidance forces. The stator is upon a small slide, whilst the rotor remains fixed centered in the stator. An axial stator misalignment is forced pulling the rotor in both directions, to measure the guidance forces that push or pull the rotor to its axial equilibrium position.

Figs. 10 and 11 compare respectively the values of lifting and guidance forces for rotors D5 and D20, obtained by experimental measurement and by FEM simulation.

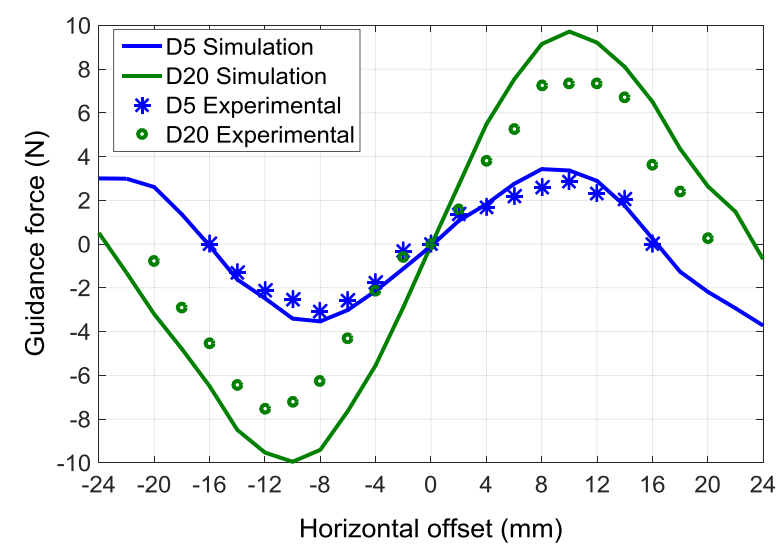

Fig. 11. Rotor guidance forces versus horizontal offset.

TABLE II

ForCES OF DisCONTINUOUS AND CONTINUOUS PM ROTOR D5

\begin{tabular}{lccccc}
\hline \hline & \multicolumn{2}{c}{ Lifting force (centered) } & & \multicolumn{2}{c}{ Guidance force } \\
\cline { 2 - 3 } \cline { 5 - 6 } Rotor D5 Weight & Simulation & Experimental & & Simulation & Experimental \\
\hline Disc. PM 10.17 N & $26.91 \mathrm{~N}$ & $18.97 \mathrm{~N}$ & & $3.12 \mathrm{~N}$ & $2.16 \mathrm{~N}$ \\
Cont. PM 15.39 N & $37.62 \mathrm{~N}$ & $30.26 \mathrm{~N}$ & & $3.43 \mathrm{~N}$ & $3.10 \mathrm{~N}$ \\
Diff. (\%) & $139.8 \%$ & $159.5 \%$ & & $109.9 \%$ & $143.5 \%$ \\
\hline \hline
\end{tabular}

The measured lifting forces are slightly lower than those estimated in the simulations. Comparing with the result obtained in [3] for the discontinuous PM rotor D5 with a $10.17 \mathrm{~N}$ weight, the levitation force is in general higher, being enough to compensate the higher weight of the $15.38 \mathrm{~N}$ weight of the continuous $\mathrm{PM}$ rotor D5.

The forces estimated show reasonable values, near to the simulated ones. In general, the forces are lower than predicted. Actually, simulation results assume ideal conditions. In practice, there are some inevitable uncertainties, namely due to liquid nitrogen leakages. This fact explains in part those discrepancies. However, the turning points, i.e., the distance at which the magnitude of guidance forces stop increasing and start decreasing, are consistent.

Table II compares the SMB force values using the old discontinuous PM rotor studied in [3]-[5] and the new continuous PM rotor using PM rings. Simulations and experimental values of lifting and guidance forces are presented.

In all cases, the forces have increased using the new rotor. Experimental results are below theoretical ones, which may be explained by inevitable liquid nitrogen losses and other experimental disturbances. However, besides those uncertainties, the both simulated and experimental values are of the same order.

\section{Dynamic Tests}

A big concern of our research team is to apply the SMB as a bearing for electrical machines. For this purpose, dynamic analysis is required to understand the viability of the SMB in operation, i.e., to understand how the system behaves when rotating. Thus, an experimental setup was installed, on one hand, to test the SMB as a bearing for electrical machines and on the 


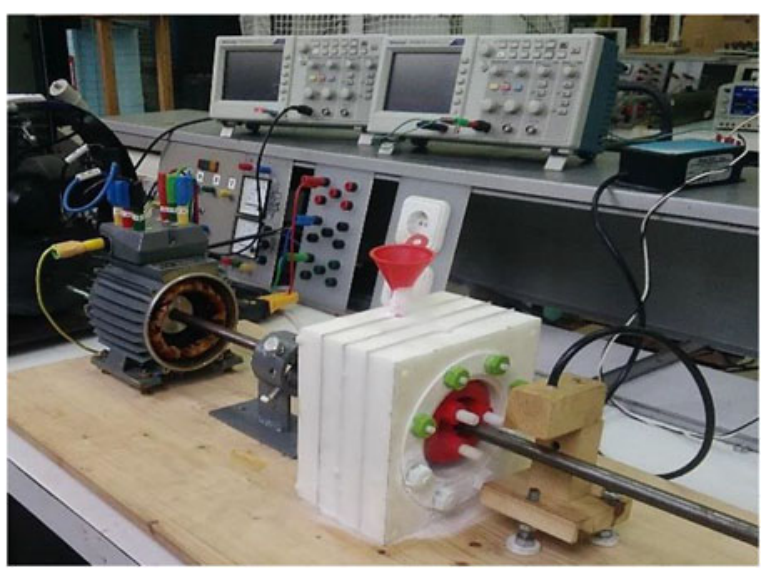

Fig. 12. Experimental setup as a bearing of an electrical machine.

TABLE III

FREQUENCIES FOR SEVERAL ANGULAR ROTATION SPEEDS

\begin{tabular}{lcccc}
\hline \hline Rotor D5 & $\begin{array}{c}f_{R} \\
(\mathrm{~Hz})\end{array}$ & $\begin{array}{c}f_{R} \\
(\mathrm{rpm})\end{array}$ & $\begin{array}{c}\text { Sensor } f_{S 1} \\
(\mathrm{~Hz})\end{array}$ & $\begin{array}{c}\text { Current } f_{C 1} \\
(\mathrm{~Hz})\end{array}$ \\
\hline Discontinuous PM rotor & 9.17 & 550 & 6.16 & 47 \\
& 14.16 & 850 & 11.96 & 50 \\
Continuous PM rotor & 4 & 240 & 4.32 & 16.4 \\
& 8.33 & 500 & 7.64 & 25 \\
& 15 & 900 & 14.61 & 40 \\
\hline \hline
\end{tabular}

other hand to compare the stability and the smoothness of the operation of the SMB using discontinuous PMs [3]-[5] and the SMB using the new continuous PM rings.

\section{A. Analysis of Vibrations When Applied to a Motor Shaft}

The main concern of the experimental test is to replace a mechanical bearing from an electrical motor, in order to understand if the SMB is able to sustain the weight of the system and to rotate in a stable way at the same time. Fig. 12 shows the used setup.

One of the mechanical bearings was removed from a two dipole three-phase motor with nominal power $370 \mathrm{~W}(1 \mathrm{Hp})$ and nominal speed $1370 \mathrm{rpm}$. The SMB was then linked to the motor rotor via a calibrated tube. The Altivar 31 frequency inverter was set to allow motor soft starts and to control the angular speed. To evaluate the stability, a high accuracy displacement sensor was set to measure oscillations and vertical offsets. The sensor is pointed to the tube. The oscillations of the system were analyzed through frequency analysis of the motor current and displacements of the tube.

Table III shows the fundamental frequencies of the supply current from the inverter and measured by the displacement sensor, for several rotor angular speeds. In this table, $f_{R}$ is the rotor frequency of rotation measured by a tachometer, $f_{S 1}$ the fundamental frequency of the signal from the displacement sensor and $f_{C 1}$ the fundamental frequency of the supply current from the inverter and the driving motor number of dipoles value is 2 .

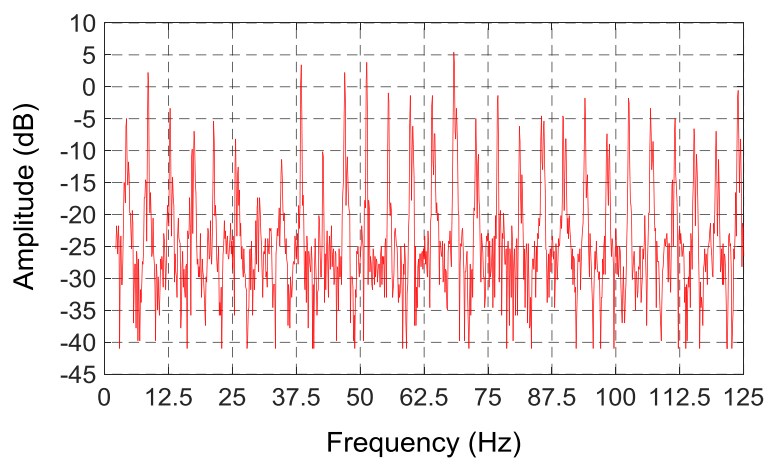

Fig. 13. Displacement sensor spectrum, for continuous rotor at $240 \mathrm{rpm}$.

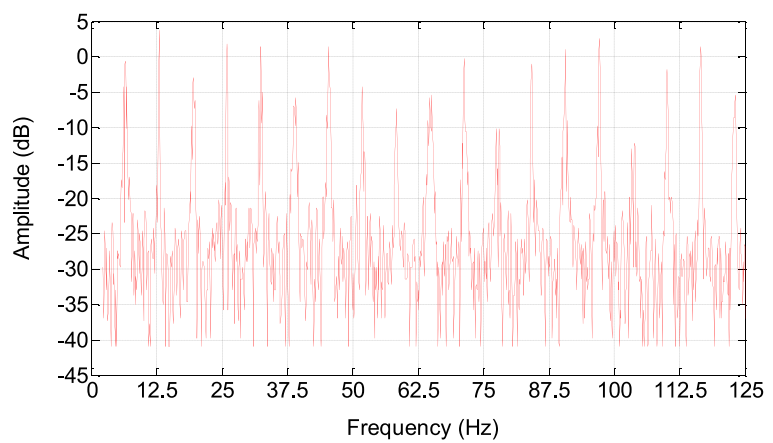

Fig. 14. Displacement sensor spectrum, for discontinuous rotor at $550 \mathrm{rpm}$.

For similar rotor speeds, the discontinuous PM rotor presented higher slip values and thus higher resistive torque than the continuous PM rotor.

For all the tested rotor speeds, the spectrum of the signal from the displacement sensor consists of a series of frequency harmonics at multiples of the fundamental frequency harmonic at $f_{S 1}$, that is almost the same as the rotor angular frequency. This corresponds in time to a series of thin pulses with period $T_{S}=1 / f_{S 1}$. This kind of spectrum is generally associated with mechanical looseness in electrical motors [15].

Because of the amplitude of vibrations and the reduced air gap, it was not possible to operate the discontinuous PM rotor at angular speeds close to $240 \mathrm{rpm}$. However, for the continuous rotor, the results are shown in Fig. 13.

Figs. 14 and 15 show the spectrum of the displacement sensor signal for the discontinuous PM rotor operating at an angular frequency of $550 \mathrm{rpm}$ and for the continuous rotor operating at an angular frequency of $500 \mathrm{rpm}$.

Figs. 14 and 15 show that, for rotor speeds around $500 \mathrm{rpm}-$ $550 \mathrm{rpm}$, the amplitude of the harmonics from the displacement sensor spectrum are generally higher for the discontinuous PM rotor.

Figs. 16 and 17 show the spectrum of the displacement sensor signal respectively for the discontinuous PM rotor at $850 \mathrm{rpm}$ and for the continuous PM rotor at $900 \mathrm{rpm}$.

For rotor speeds between $850 \mathrm{rpm}$ and $900 \mathrm{rpm}$, the amplitude of the harmonics from the displacement sensor spectrum is also generally higher for the discontinuous PM rotor. 


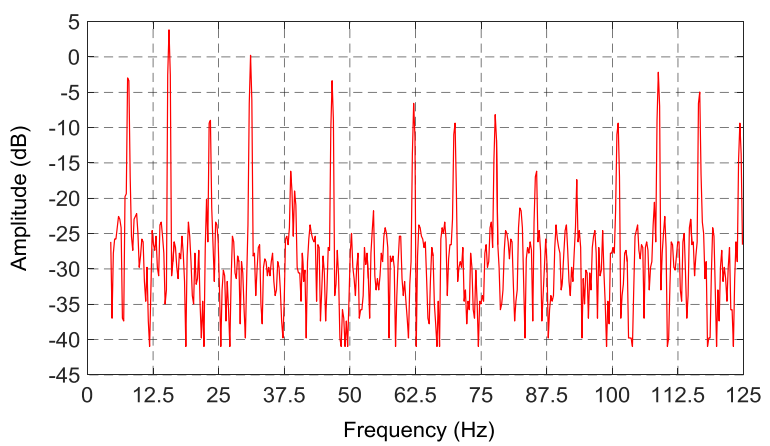

Fig. 15. Displacement sensor spectrum, for continuous rotor at $500 \mathrm{rpm}$.

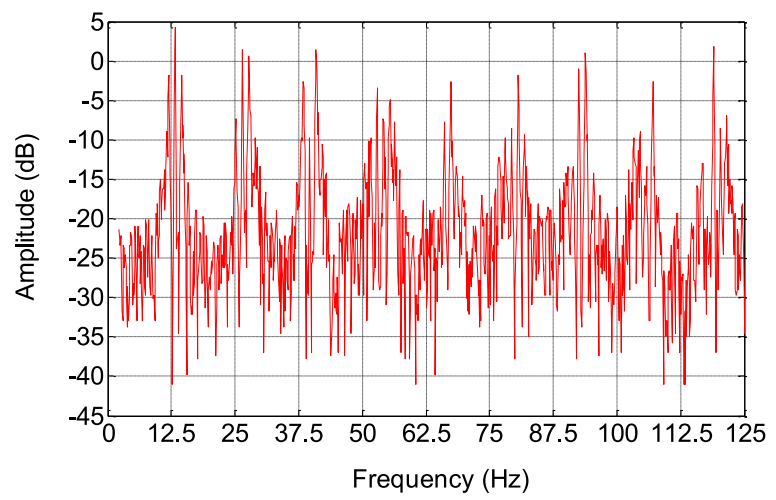

Fig. 16. Displacement sensor spectrum, for discontinuous rotor at $850 \mathrm{rpm}$.

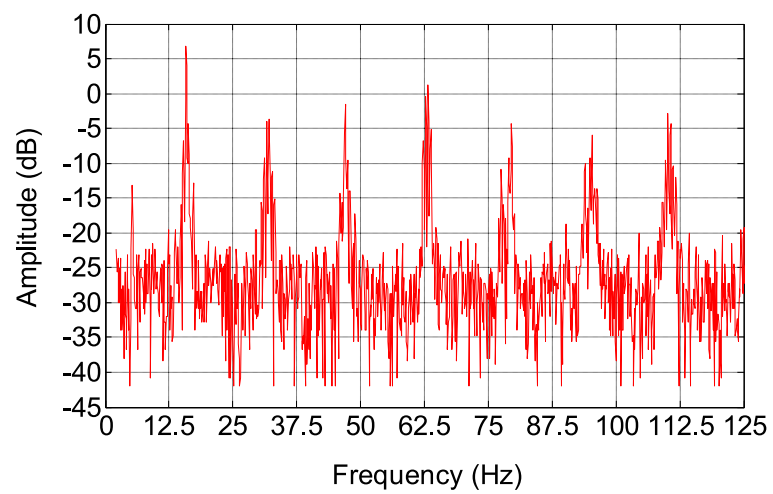

Fig. 17. Displacement sensor spectrum, for continuous PM rotor at $900 \mathrm{rpm}$.

\section{B. Radial Dynamics}

Fig. 18 shows the setup used to evaluate the radial dynamics of the system. The shaft is vertically moved in the opposite direction of the sensor to the position where the rotor touches the stator, which is $5 \mathrm{~mm}$. The shaft is then dropped and the change of position with time is measured by a fiber optic displacement sensor (PHILTEC, Inc.) with a sensibility of $0.5 \mathrm{mV} / \mu \mathrm{m}$ and with a resolution of $7 \mu \mathrm{m}$.

The radial dynamics of the system are modeled approximately by the transfer function presented in (1):

$$
G_{r}(s)=\frac{1811}{s^{2}+1.019 s+1746}
$$

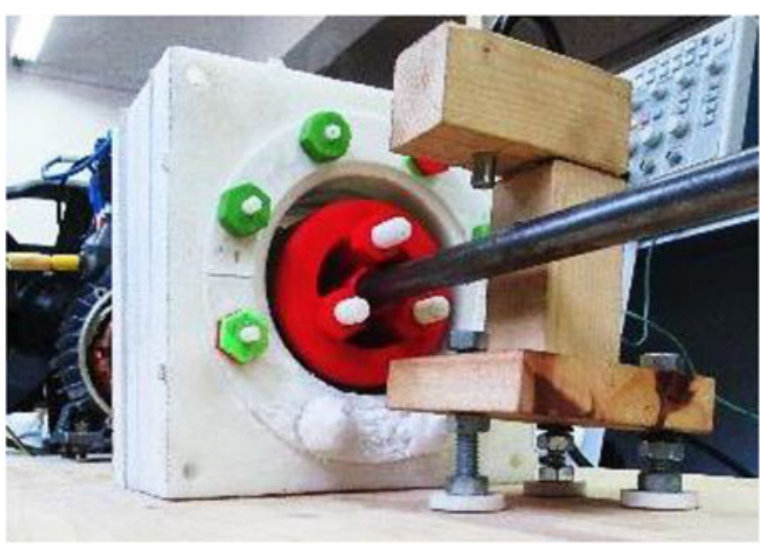

Fig. 18. Experimental setup used to evaluate the radial dynamics.

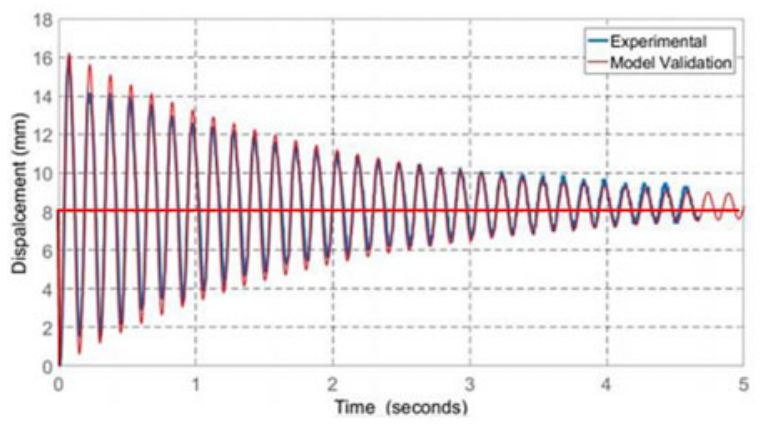

Fig. 19. Experimental data and model validation for rotor vertical offset response.

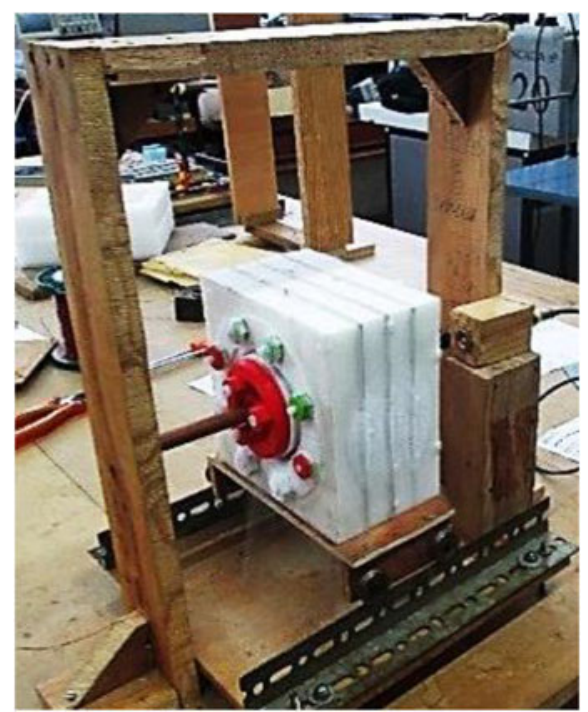

Fig. 20. Experimental setup used to acquire the axial dynamics.

that defines a 2 nd order system, where $s$ is the undetermined Laplace Transform variable.

Fig. 19 shows the rotor vertical offset response to an $8 \mathrm{~mm}$ amplitude step. 


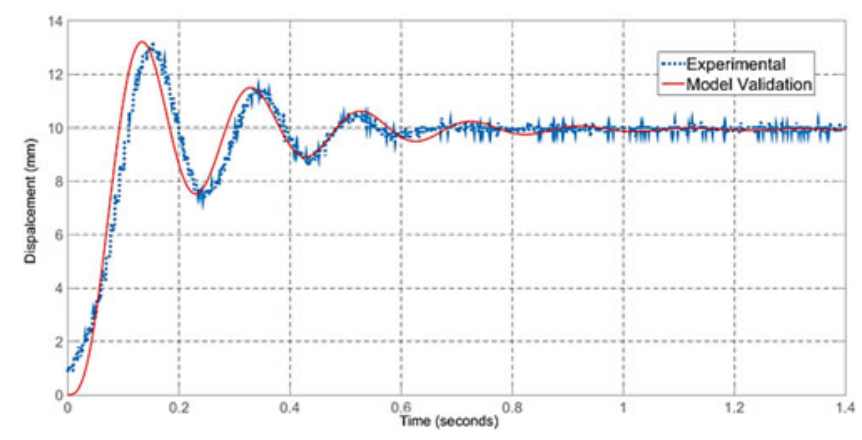

Fig. 21. Experimental data and model validation for axial system response.

\section{Axial Dynamics}

Fig. 20 shows the setup used to acquire the response curve of the system when excited with a step. The stator is displaced to the point where the guidance forces are maximum, which is around $10 \mathrm{~mm}$, and dropped. The position is measured by a position sensor as a function of time.

The axial dynamics of the system are modeled approximately by the transfer function presented in (2):

$$
G_{r}(s)=\frac{3.47 \times 10^{4}}{s^{3}+41.06 s^{2}+1365 s+3.49 \times 10^{4}}
$$

that defines a 3 rd order system.

Fig. 21 shows the obtained response curve and the simulation of the model (2) response for a $10 \mathrm{~mm}$ step input.

The initial overshoot is relatively high and there are oscillations in the response. A better response will be achieved by active controllers and will be subject to further work.

\section{CONCLUSION}

Notable advances were achieved using a rotor based on continuous ring PMs in the prototype. In fact, the rotation of the SMB with the continuous ring is much smoother and with fewer harmonics than the version with the discontinuous rotor.

FEM models were revealed to be well developed and the lifting and guidance forces experimentally measured are in the same order of magnitude as the predictions. However, one can note that the discrepancies between simulated and experimental forces are higher for the discontinuous rotor. Let's analyze first the continuous and the discontinuous rotor D5 solutions. When measuring the guidance forces in the discontinuous solution, there were two superconductors that, although intact, presented a higher state of degradation (cracked) when compared to the other four ones. Furthermore, in the discontinuous rotor D5, the PMs were repelled outwardly, their faces not being perfectly perpendicular to the radial direction. In conclusion, these two drawbacks caused guidance forces from simulations to be overestimated more for discontinuous than continuous PM rings for rotor D5.

The continuous rotor D5 solution was built using two new superconductor bulks. Without cracks perturbing the magnetic field distribution, differences between the measured and simulated guidance forces became smaller. However, in the continuous rotor D20, simulated and measured forces showed higher differences. In the continuous rotor D20 solution, since the PM rings are further apart from each other than in the continuous rotor D5, the magnetic flux passing between the magnets is higher. Therefore, differences in relation to the accuracy of the magnetic field distribution produced greater discrepancies in the calculation of guidance forces in D20.

The increase in lifting forces compensates the rotor weight increase well, allowing a lower diameter rotor and higher air gap distance, improving the operational security.

Also, lifting forces and guidance forces can be adjusted by the spacing between PM rings: rotor D5 exhibits higher lifting forces and rotor D20 higher guidance forces.

As for dynamic analysis, it was shown that the SMB with a continuous PM ring rotor out-performs the discontinuous PM rotor in all ranges of frequency tested.

\section{REFERENCES}

[1] P. J. Costa Branco and J. A. Dente, "Design and experiment of a new maglev design using zero-field-cooled YBCO superconductors," IEEE Trans. Ind. Electron., vol. 59, no. 11, pp. 4120-4127, Nov. 2012.

[2] J. Fernandes, I. Montes, R. Sousa, C. Cardeira, and P. J. Costa Branco, "Superconductor Joule losses in a zero-field-cooled (ZFC) maglev vehicle," IEEE Trans. Appl. Supercond., vol. 26, no. 3, Apr. 2016, Art. no. 8201907.

[3] A. J. Arsénio, M. V. Carvalho, C. Cardeira, R. Melicio, and P. J. Costa Branco, "Experimental setup and efficiency evaluation of zero-fieldcooled (ZFC) YBCO magnetic bearings," IEEE Trans. Appl. Supercond., vol. 27, no. 4, Jun. 2017, Art. no. 3601105.

[4] A. J. Arsénio, M. V. Carvalho, C. Cardeira, R. Melicio, and P. J. Costa Branco, "Conception of a YBCO superconducting ZFC-magnetic bearing virtual prototype," in Proc. IEEE Int. Power Electron. Motion Control Conf., Varna, Bulgaria, Sep. 2016, pp. 1226-1230.

[5] A. J. Arsénio, M. V. Carvalho, C. Cardeira, R. Melicio, and P. J. Costa Branco, "Viability of a frictionless bearing with permanent magnets and HTS bulks," in Proc. IEEE Int. Power Electron. Motion Control Conf., Varna, Bulgaria, Sep. 2016, pp. 1231-1236.

[6] M. Sparing et al., "Dynamics of rotating superconducting magnetic bearings in ring spinning," IEEE Trans. Appl. Supercond., vol. 26, no. 3, Apr. 2016, Art. no. 3600804.

[7] S. Sivrioglu, S. Basaran, and A. Yildiz, "Multi surface HTS-PM levitation for a flywheel system," IEEE Trans. Appl. Supercond., vol. 26, no. 8, Oct. 2016, Art. no. 3603206.

[8] Z. Yu, G. Zhang, Q. Qiu, L. Hu, B. Zhuang, and M. Qiu, "Analyses and tests of HTS bearing for flywheel energy system," IEEE Trans. Appl. Supercond., vol. 24, no. 3, Jun. 2014, Art. no. 5700405.

[9] S. Sivrioglu and S. Basaran, "A dynamical stiffness evaluation model for a ring-shaped superconductor magnetic bearing system," IEEE Trans. Appl. Supercond., vol. 25, no. 4, Aug. 2015, Art. no. 3601507.

[10] S. Sakai, Q. Koichi, and O. Shunsuke, "Effect of the magnetic configuration on the rotational motion in the attractive-type HTS-permanent magnet hybrid bearing," IEEE Trans. Appl. Supercond., vol. 26, no. 4, Jun. 2016, Art. no. 3601204.

[11] J. Arnaud and P. J. Costa Branco, "Electrothermal characteristics of YBCO bulk magnets deep in LN2: A preliminary analysis for its use as excitation system of low-speed synchronous generators," IEEE Trans. Appl. Supercond., vol. 26, no. 3, Apr. 2016, Art. no. 6800608.

[12] Can Superconducters, Superconducting Ybco levitation bulk. [Online] Available: https://www.can-superconductors.com/uploads/2/1/2/9/2129 8520/csyl_092017.pdf. Accessed on: Jan. 19, 2018.

[13] T. Nakagawa, T. Nakiri, R. Hosoya, and Y. Tajitsu, "Electrical properties of biodegradable polylactic acid film," IEEE Trans. Ind. Appl., vol. 40, no. 4, pp. 1020-1024, Jul./Aug. 2004.

[14] J. Drobny, "Properties of polytetrafluoroethylene," in Specialty Thermoplastics. Berlin, Germany: Springer, 2015, pp. 5-9.

[15] J. Mais, Spectrum Analysis: The Key Features of Analyzing Spectra. San Diego, CA, USA: SKF, May 2002. 